\title{
Statistical model for the structure and gelation of smectite clay suspensions
}

\author{
Marjolein Dijkstra, ${ }^{*}$ Jean-Pierre Hansen ${ }^{\dagger}$ and Paul A. Madden \\ Physical and Theoretical Chemistry Laboratory, University of Oxford, South Parks Road, Oxford OX1 3QZ, United Kingdom
}

(Received 13 June 1996)

\begin{abstract}
The sol-gel transition in a suspension of monodisperse, charged, disklike platelets (Laponite, i.e., a synthetic clay) is examined within a simplified statistical model. The initial "primitive" model of uniformly charged disks surrounded by microscopic co ions and counterions is reduced to a model of nonintersecting disks carrying a rigid point quadrupole, resulting from the electric double layers around the disks. The quadrupolar interactions favor edge-to-face pair configurations that counteract the tendency of parallel (nematic) alignment of bare disks at high densities. The local structure and phase behavior of the quadrupolar disk model is studied over a broad range of clay concentrations and quadrupole moments (which depend on the concentration of added salt) by extensive Monte Carlo simulations. The model suspension is found to undergo a reversible sol-gel transition above a critical quadrupolar coupling. The gel phase lacks long-range order, and is reminiscent of a "house-of-cards" structure in which most of the particles are edge-to-face to each other. The critical concentration and quadrupolar coupling constant are not inconsistent with recent experimental data on the gelation of Laponite suspensions. [S1063-651X(97)10502-5]
\end{abstract}

PACS number(s): 82.70.Gg, 61.20.Gy, 64.70.-p

\section{INTRODUCTION}

Smectite clays of the montmorrilonite group are silicate minerals (phyllosilicates) with a layered structure. In dry clays the spacing between layers along the $c$ axis is typically $1 \mathrm{~nm}$. In the presence of water, this spacing increases due to the adsorption of water molecules between the layers, leading to the phenomenon of swelling. Each individual lamella (or platelet) carries a negative surface charge, due to isomorphous substitution of polyvalent cations $\left(\mathrm{Si}^{4+}\right.$ or $\left.\mathrm{Al}^{3+}\right)$ by cations of lower valence $\left(\mathrm{Al}^{3+}\right.$ or $\left.\mathrm{Mg}^{2+}\right)$ in the crystalline layers. Counterions (generally $\mathrm{Na}^{+}$), and ions from added salt, then form electric double layers around the lamellae, which are the origin of the strong electrostatic interactions between clay platelets.

With increasing water content, the swollen clay turns into a gel of entangled, flexible lamellae, the complex rheological behavior of which is the basis of important technological applications, such as oil drilling.

In the highly dilute regime, when the concentration by weight of clay in water drops to a few percent, an isotropic colloidal dispersion of clay platelets is obtained, where the latter are still correlated via the long-range Coulombic interactions, but orientational ordering of the platelets is completely lost. The transition between the dilute sol phase and the more concentrated gel is sensitive to the concentration of added salt and appears to be reversible [1]. The microstructure of the gel has been explored by a number of recent small-angle neutron-scattering [2], $\mathrm{x}$-ray scattering [1,3], and light-scattering [4] experiments. The slowing down of con-

\footnotetext{
*Present address: Shell Research and Technology Centre Amsterdam, Badhuisweg 3, 1031 CM Amsterdam, The Netherlands.

${ }^{\dagger}$ On leave of absence from Laboratoire de Physique, Unité de Recherche Associée 1325 du Centre National de la Recherche Scientifique, Ecole Normale Supeérieure de Lyon, 69364 Lyon Cedex 07, France.
}

centration fluctuations at the sol-gel transition has been analyzed by dynamic light scattering [5]. Despite these careful experimental investigations, the microstructure of the gel, and the individual and collective motions of the platelets, which are intimately related to the macroscopic rheological behavior of concentrated clay colloid dispersions, are not fully understood. The small-angle neutron and x-ray data favor a heterogeneous microstructure, involving microdomains of variable platelet concentration, over the traditional "house-of-cards" structure, dominated by edge-to-face configurations of neighboring platelets [6]. It hence seems desirable to supplement the experimental data by theoretical calculations based on a reasonable model of clay particles in suspension. This is a very difficult task for natural clay suspensions involving irregularly shaped, bent, and highly polydisperse lamellae. Fortunately, synthetic clays have become available, like Laponite RD [7], which have a crystalline unit cell rather similar to that of natural montmorillonites, but are made up of nearly monodisperse, thin cylindrical platelets. A typical Laponite particle has the appearance of a flat coin of diameter $\sigma \simeq 25 \mathrm{~nm}$, thickness $d \simeq 1 \mathrm{~nm}$, and carries a charge of $-0.7 e$ per unit cell, amounting to a total charge of roughly $-700 e$.

Since the lateral dimension of a Laponite platelet is an order of magnitude smaller than that of natural clay lamellae, the platelet may be regarded as being rigid (i.e., nonbending). A suspension of such monodisperse, coinlike charged particles is clearly more amenable to theoretical modeling than natural clays and this paper presents an attempt at a statistical description of Laponite suspensions, aimed at exploring the osmotic equation of state, the local structure and the sol-gel transition, as functions of the two fundamental control parameters, i.e., the particle concentration and the ionic strength of the suspension. The main practical problem in any first principles description of colloidal systems such as clay dispersions is how to overcome the considerable asymmetry between the highly charged, mesoscopic polyions and the microscopic coions and counterions. 
In Sec. II, we formulate a density functional approach to the "primitive" model of Laponite suspensions. In view of the considerable technical difficulties associated with the anisometry of the lamellar polyions, the theoretical reduction process will be taken one step further in Sec. III, where the quadrupolar disk model is introduced. Monte Carlo simulations of this model are described in Sec. IV. The main results from these simulations, including the location of the sol-gel transition, are presented and discussed in Sec. V, and some concluding remarks are made in Sec. VI. A preliminary account of some of this work has been given elsewhere [7].

\section{THE “PRIMITIVE' MODEL OF LAPONITE SUSPENSIONS}

The "primitive" model under consideration is an extension of the common model of ionic solutions. $N$ infinitely thin, uniformly charged disks of diameter $\sigma$ and total charge $-Z e$ are placed in a volume $V$, together with $N Z$ monovalent counterions and salt, at a concentration $n_{s}$ per unit volume. The molecular nature of the solvent is ignored, and water replaced by a structureless continuum of dielectric constant $\epsilon$ (it is this simplification that earns the model its unflattering designation as "primitive"). The disks, which rotate freely in space, are not allowed to intersect, and the neglect of the finite thickness of the Laponite platelets is justified by their considerable aspect ratio $\sigma / d \simeq 25$. The uniform surface charge density of a disk will be denoted by $q=-Z e / \pi(\sigma / 2)^{2}$, and the Laponite concentration by $n=N / V$. Room temperature and strict monodispersity in charge and size of the platelets will be assumed throughout. The monovalent positive counterions and negative coions are regarded as charged point particles. To avoid Coulomb collapse a cutoff in the divergent attraction between opposite point charges of the order of a typical ionic diameter must be introduced; no such cutoff is needed between ions of equal charge, and between counterions and uniformly charged platelets. The independent thermodynamic variables, besides the fixed temperature $T$, are the platelet and salt concentrations $n$ and $n_{s}$; although in the present model the infinitely thin platelets have zero volume, the nonintersection condition makes it convenient to introduce the dimensionless density variable $n^{*}=n \sigma^{3}$; if the normals to all disks were perfectly aligned (perfect nematic order), there would be no limit to the packing capacity, i.e., $n^{*}$ could, in principle, grow to infinity. In practice any deviation from perfect alignment, and the Coulomb repulsion between disks will limit the range of variation of $n^{*}$. Despite its apparent simplicity, the model will lead to two major difficulties in any attempt of explicit theoretical calculations. The first complication is of an essentially technical nature. Due to the highly anisometric shape of the polyions, Gauss' theorem does not apply and the total Coulomb potential between two platelets, say $i$ and $j$, is a complicated function of their center-of-mass positions $\vec{R}_{i}$ and $\vec{R}_{j}$ and of their orientations, materialized by the unit vectors $\vec{n}_{i}$ and $\vec{n}_{j}$ normal to the platelets; this function may be expressed as a multiple integral over the surfaces of the two interacting platelets, which cannot be explicitly evaluated, even for very simple configurations. This technical difficulty can be overcome by numerical means, e.g., by discretizing the surface charge on the platelets, but at the cost of considerable computational expense.

The second complication is of a more fundamental nature, and is related to the considerable polyion-microion asymmetry, due to the large ratios in size and time scales associated with the mesoscopic and microscopic species. This makes a first-principles description, where the two species would be treated on an equal footing, untractable, particularly in conjunction with computer simulations. This difficulty may, however, be overcome by adopting complementary points of view for the two populations: the polyions will be considered as discrete individual particles, whereas a coarse-grained description is adopted for the microions, which will not be treated individually, but rather at the level of the instantaneous local densities $\rho_{+}(\vec{r})$ and $\rho_{-}(\vec{r})$ of the counterions and coions. Due to the large difference in time scales, the microions may be assumed to be distributed in thermodynamic equilibrium around the polyions, for any configuration $\left\{X_{i}\right\}=\left\{\vec{R}_{i}, \vec{n}_{i}\right\}(1 \leqslant i \leqslant N)$ of the latter (adiabatic approximation). This allows a free energy functional $\mathcal{F}\left[\rho_{+}(\vec{r}), \rho_{-}(\vec{r})\right]$ to be defined, which depends parametrically on the $\left\{X_{i}\right\}$. According to the basic principles of density functional theory (DFT) [8], the local densities may be determined for any given polyion configuration $\left\{X_{i}\right\}$, by the variational principle

$$
\frac{\partial \mathcal{F}\left[\rho_{+}, \rho_{-}\right]}{\partial \rho_{\alpha}(\vec{r})}=\mu_{\alpha}, \quad \alpha=+,-,
$$

where $\mu_{\alpha}$ is the Lagrange multiplier associated with the constraint

$$
\int_{V} \rho_{\alpha}(\vec{r}) d \vec{r}=N_{\alpha}, \quad \alpha=+,-,
$$

i.e., the chemical potential associated with species $\alpha$. The functional $\mathcal{F}$ may be split into the usual ideal, Coulombic, external, and correlational contributions [9]:

$$
\begin{gathered}
\mathcal{F}=\mathcal{F}_{\text {id }}+\mathcal{F}_{\text {Coul }}+\mathcal{F}_{\text {ext }}+\mathcal{F}_{\text {corr }}, \\
\left.\mathcal{F}_{\text {id }[}\left\{\rho_{\alpha}\right\}\right]=k_{B} T \sum_{\alpha=+,-} \int_{V} d \vec{r} \rho_{\alpha}(\vec{r})\left\{\ln \left[\Lambda_{\alpha}^{3} \rho_{\alpha}(\vec{r})\right]-1\right\} \\
\mathcal{F}_{\text {Coul }}\left[\left\{\rho_{\alpha}\right\}\right]=\frac{e^{2}}{2 \epsilon} \int_{V} \int_{V} d \vec{r} d \overrightarrow{r^{\prime}} \frac{\rho_{c}(\vec{r}) \rho_{c}\left(\overrightarrow{r^{\prime}}\right)}{\left|\vec{r}-\overrightarrow{r^{\prime}}\right|}, \\
\mathcal{F}_{\text {ext }}\left[\left\{\rho_{\alpha}\right\}\right]=\frac{e}{\epsilon_{i=1}^{N}} \int_{V} d \vec{r} \rho_{c}(\vec{r}) \varphi\left(\vec{r} ; X_{i}\right), \\
\left.\mathcal{F}_{\text {corrL }}\left\{\rho_{\alpha}\right\}\right] \simeq \int_{V} d \vec{r} \rho(\vec{r}) f_{\text {exc }}\left(\rho_{+}(\vec{r}), \rho_{-}(\vec{r})\right)
\end{gathered}
$$

In these equations, $\rho(\vec{r})=\rho_{+}(\vec{r})+\rho_{-}(\vec{r}) \quad$ and $\rho_{c}(\vec{r})=\rho_{+}(\vec{r})-\rho_{-}(\vec{r})$ denote the local number and charge densities of the microions, $\Lambda_{\alpha}$ is the thermal de Broglie wavelength of species $\alpha$, and $\varphi\left(\vec{r}, X_{i}\right)$ is the electrostatic potential at $\vec{r}$, due to the $i$ th uniformly charged disk centered at $\vec{R}_{i}$ with orientation $\vec{n}_{i}$. For the correlational part, we have adopted the local density approximation (LDA), where 
$f_{\text {exc }}\left(\rho_{+}, \rho_{-}\right)$is the excess (nonideal) free energy per ion of a homogeneous "primitive" solution of coions and counterions with (uniform) densities $\rho_{-}=\rho_{-}(\vec{r})$ and $\rho_{+}=\rho_{+}(\vec{r})$; in order for $f_{\text {exc }}$ to be defined in the thermodynamic limit, charge neutrality of this homogeneous solution requires the presence of a uniform neutralizing background of charge density $-\rho_{c}=-\rho_{c}(\vec{r})$.

Once the $\rho_{\alpha}(\vec{r})$ have been determined by inserting the expressions of Eq. (3) into Eq. (1), the effective interaction energy between the polyions is given by

$$
V_{\text {eff }}\left(\left\{X_{i}\right\}\right)=\sum \sum_{i<j} v_{p}\left(X_{i}, X_{j}\right)+\mathcal{F}\left[\left\{\rho_{\alpha}\right\} ;\left\{X_{i}\right\}\right]
$$

where $v_{p}\left(X_{i}, X_{j}\right)$ is the direct (Coulomb) interaction potential between polyions $i$ and $j$, and $\mathcal{F}$ is the minimized free energy, a functional of the optimum density profiles for the given polyion configuration $\left\{X_{i}\right\}$; this second contribution to the polyion interaction energy is not, in general, pairwise additive, but depends on the coordinates of all polyions (many-body interaction).

In practice, the minimization [Eq. (1)] may be carried out numerically, by steepest descent or conjugate gradient methods, and the resulting free energy, combined with the direct Coulomb potential energy between polyions, may then be used as input in standard Monte Carlo simulations of samples of a few hundred polyions. Such a procedure has already been successfully applied to linear polyelectrolytes (charged polymer chains) [10]. Alternatively, the minimization may be carried out dynamically, within an adiabatic molecular dynamics scheme similar in spirit to the "ab initio" method of Car and Parrinello for ion-electron systems [11]. The effective forces and torques between polyions are calculated "on the fly" via the classical version of the HellmannFeynman theorem. This method has been put to use to simulate concentrated suspensions of charged spherical colloidal particles [9]. The direct application of the method to clay suspensions is hampered by the difficulty of calculating efficiently the Coulomb energy between two disks in an arbitrary configuration as stressed earlier. For practical calculations, further approximations are needed. We have adopted the following two-step strategy: (a) The initial $N$-polyion problem is reduced to a one-polyion problem by confining a single uniformly charged disk and the associated coions and counterions to a Wigner-Seitz (WS) cell of volume $v=V / N=1 / n$; the WS cell is electrically neutral and is meant to be a crude representation of the "caging" of a given platelet by its neighbors. For sufficiently dilute suspensions (sol phase), where the platelets may be regarded as freely rotating, it is not unreasonable to assume the WS cell to be a sphere of radius $R=(3 v / 4 \pi)^{1 / 3}$. For very high concentrations (swelling regime), where the platelets are roughly parallel, a cylindrical WS cell is more appropriate. The anisotropic density profiles of the coions and counterions may then be determined by solving the variational problem [Eq. (1)] with an external field contribution $\mathcal{F}_{\text {ext }}$ due to a single polyion placed at the center of the WS cell, under appropriate boundary conditions at the surface of the cell. This program has been carried out for spherical and cylindrical WS cells, in the linearized Poisson-Boltzmann approximation, which amounts to neglecting $\mathcal{F}_{\text {corr }}$, and to replacing $\mathcal{F}_{\text {id }}$ by its functional expansion to second order in $\Delta \rho_{\alpha}(\vec{r})$ $=\rho_{\alpha}(\vec{r})-n_{\alpha}[12]$. The resulting density profiles have been used to compute the quadrupole moment of the total charge distribution within the WS cell, i.e., the sum of contributions from the uniform charge density on the disk $\left(Q_{d}\right)$ and from the coion and counterion density profiles $\left(Q_{c}\right)$ (the electric dipole moment vanishes since the total charge distribution is centrosymmetric). If the $z$-axis is chosen along the normal $\vec{n}$ to the disk, the quadrupole tensor $\mathbf{Q}$ may be expressed as

$$
\begin{gathered}
\mathbf{Q}=\frac{Q}{2}(3 \vec{n} \vec{n}-\mathbf{I}) \\
Q=Q_{d}+Q_{c} \\
Q_{d}=-Z e \frac{\sigma^{2}}{16} \\
Q_{c}=\frac{e}{2} \int_{v} \rho_{c}(\vec{r})\left(3 z^{2}-r^{2}\right) d \vec{r}
\end{gathered}
$$

Since $Q_{d}$ and $Q_{c}$ are of opposite sign, the ratio $\alpha=Q_{\text {total }} / Q_{d}=\left(Q_{c}+Q_{d}\right) / Q_{d}$ is always less than one, but depends on the state variables $n$ and $n_{s}$. (b) The second step in the present strategy is to return now to the initial $\mathrm{N}$-polyion problem, replacing each polyion and its associated electric double layer of coions and counterions by an "effective" polyion which is electrically neutral, but carries a fixed quadrupole moment $Q$, which is coupled to the quadrupole moments of all other polyions by the usual laws of electrostatics. The initial system of polyions and microions has thus been reduced to a model of quadrupolar disks to which we turn in the following section. The limitations of the model are obvious: all higher order multipole moments are neglected; the interference between double layers associated with different polyions is ignored, which is certainly a poor approximation whenever two polyions come very close, i.e., when there is considerable overlap between their associated double layers; such an overlap will break the symmetry of the charge distribution around an isolated platelet, and hence lead to induced dipoles and changes in the instantaneous higher order multipoles. On the contrary, in the quadrupolar disk model, the electric double layer is assumed to be completely rigid (nonpolarizable), independently of the charge distributions of the surrounding polyions. The model is thus based on a mean-field assumption at the level of the electric double layers.

\section{THE QUADRUPOLAR DISK MODEL}

The reduction procedure sketched in Sec. II leaves us with a system of identical "effective" polyions that reduce to infinitely thin disks of diameter $\sigma$, carrying a constant axial quadrupole moment $Q$. The total potential energy for allowed configurations (i.e., without intersecting disks) is the pair-wise sum of quadrupolar interactions:

$$
V_{N}\left(\left\{X_{i}\right\}\right)=\sum \sum_{i<j} v_{Q Q}\left(X_{i}, X_{j}\right)
$$




$$
v_{Q Q}\left(X_{i}, X_{j}\right)=-\frac{1}{9} \mathbf{Q}_{i}: \mathbf{T}_{\mathbf{i j}}^{(4)}: \mathbf{Q}_{j}
$$

where

$$
\mathbf{T}_{i j}^{(4)}=-\nabla_{i}^{(4)} \frac{1}{\epsilon\left|\vec{R}_{i}-\vec{R}_{j}\right|}
$$

is the quadrupolar tensor; if $\vec{R}_{i j}=\vec{R}_{i}-\vec{R}_{j}$, Eq. (10) may be cast in the form [13]

$$
v_{Q Q}\left(\vec{R}_{i j} ; \vec{n}_{i}, \vec{n}_{j}\right)=\frac{3 Q^{2}}{4 \epsilon R_{i j}^{5}} \Phi^{224}(i, j),
$$

where the rotational invariant $\Phi^{224}$ depends on the relative orientations of the center-to-center vector $\vec{R}_{i j}$ and of the orientation vectors $\vec{n}_{i}$ and $\vec{n}_{j}$. If $\vec{R}_{i j}$ is chosen as the polar axis for the orientations $\vec{n}_{i}$ and $\vec{n}_{j}$, specified by the polar angles $\Omega_{i}=\left(\theta_{i}, \varphi_{i}\right)$ and $\Omega_{j}=\left(\theta_{j}, \varphi_{j}\right), \Phi^{224}(i, j)$ reduces to

$$
\begin{aligned}
\Phi^{224}(i, j)= & 1-5 \cos ^{2} \theta_{i}-5 \cos ^{2} \theta_{j}-15 \cos ^{2} \theta_{i} \cos ^{2} \theta_{j} \\
& +2\left[\sin \theta_{i} \sin \theta_{j} \cos \left(\varphi_{i}-\varphi_{j}\right)-4 \cos \theta_{i} \cos \theta_{j}\right]^{2}
\end{aligned}
$$

$\Phi^{224}$ is positive (and hence the electrostatic interaction repulsive) when disks are coaxial, so that discotic stacking is energetically unfavorable as soon as $Q \neq 0$. On the other hand $\Phi^{224}$ is negative (i.e., the electrostatic interaction is attractive) for T-shaped (edge-to-face) pair configurations, which are energetically the most favorable. When the disks are parallel but their normals make an angle around $\pi / 4$ with $\vec{R}_{i j}, \Phi^{224}$ is also negative, and since the disks do not intersect, the electrostatic attraction blows up like $1 / R_{i j}^{5}$. This unphysical electrostatic collapse is a consequence of the representation of a spatially extended charge distribution (the electric double layer) by a point quadrupole. To circumvent this unpleasant feature of the model, an infinite repulsive barrier for center-to-center distances $R \leqslant \sigma / 2$ is added. This barrier does not affect the energetically favorable $\mathrm{T}$-shaped pair configuration, which will turn out to play a central role in the formation of a gel network. Note that these hard cores limit the maximum reduced density of disks, to $n^{*}=n \sigma^{3}=48 \eta_{c p} / \pi \simeq 11.3$, where $\eta_{c p}=\pi \sqrt{2} / 6$ is the maximum fcc packing fraction of spheres.

A thermodynamic state of the system of quadrupolar disks may be characterized by two dimensionless variables, i.e., $n^{*}$ and the quadrupolar, coupling constant

$$
\beta^{*}=\frac{Q^{2}}{\epsilon k_{B} T \sigma^{5}}
$$

The mapping of the initial problem of polyions plus counterions plus salt onto the system of quadrupolar disks has reduced the number of thermodynamic variables from three $\left(n, n_{s}\right.$, and $\left.T\right)$ to two $\left(n^{*}\right.$ and $\left.\beta^{*}\right)$. In fact $\beta^{*}$ depends both on $T$ and on $n$ and $n_{s}$ (through the effective quadrupolar moment $Q)$. Varying the salt concentration amounts to changing $Q$ and hence $\beta^{*}$. Regarding $n^{*}$ and $\beta^{*}$ as independent variables amounts to neglecting the $n$ dependence of
$Q$; the Wigner-Seitz calculations of Ref. [12] show that this is not an unreasonable assumption, at least at fairly high polyion concentrations $\left(n^{*} \geqslant 1\right)$.

The case of bare disks $(Q=0)$, corresponding to the limit $\beta^{*}=0$ of the present model, has been examined in detail by Eppenga and Frenkel [14]. Their Monte Carlo (MC) simulations revealed a first-order phase transition from an isotropic fluid to a nematic phase, where the orientation vectors $\vec{n}_{i}$ align on average, to allow a more efficient packing of the disks; the transition takes place when $n^{*} \simeq 4$. While the tendency to nematic ordering is enhanced by the addition of an electric dipole moment, parallel to the orientation vectors $\vec{n}_{i}$ of the disks, which may lead to columnar phases [15], such a tendency is counteracted by quadrupolar interactions, which favor T-shaped configurations as noted earlier. Thus a nonzero value of the quadrupole moment $Q$ will frustrate the tendency of the bare disks to stack parallel to each other at high polyion concentrations.

\section{MONTE CARLO CALCULATIONS}

To gain insight into the consequences of the competition between conflicting electrostatic and stacking tendencies, we have carried out MC simulations in which the quadrupole coupling parameter $\beta^{*}$ was progressively increased. Along a given "isotherm," corresponding to a fixed value of $\beta^{*}$, the equation of state, i.e., the osmotic pressure $\Pi$ of the polyions, was calculated as a function of $n^{*}$ in a single simulation, by "'measuring" the sedimentation profile $\rho(z)$ of a suspension subjected to a gravitational potential $\phi(z)=-m g z$ in the $z$ direction; periodic boundary conditions were imposed in the $x$ and $y$ directions. The equilibrium sedimentation profile, coarse grained on a scale of the order of the disk diameter $\sigma$, satisfies the osmotic equilibrium condition

$$
\frac{d \Pi(z)}{d z}=\frac{d \Pi(\rho(z))}{d z}=-m g \rho(z),
$$

where the assumption of local thermodynamic equilibrium is made, i.e., for a fixed value of $\beta^{*}$, $\Pi$ depends on altitude only via the local density $\rho(z)$. The density profile can be accurately determined as a function of $z$ in a MC simulation, and a numerical integration of Eq. (14) then yields the osmotic equation of state $\Pi^{*}\left(n^{*}\right)=\Pi\left(n^{*}\right) \sigma^{3} / k_{B} T$ [16-18]. This procedure avoids a tedious and time-consuming pointby-point construction of the equation of state from simulations carried out on a homogeneous suspension for several densities $n^{*}$, while ensuring comparable statistical accuracy.

The results of our calculation for $\beta^{*}=0$ are compared in Fig. 1 to the data of Eppenga and Frenkel [14]. The agreement is found to be excellent, and independent of the strength of the applied gravitational field, measured by the dimensionless ratio $\alpha=m g \sigma / k_{B} T$, as it should. The discrepancies observed for $n^{*} \geqslant 4$ are not surprising, since the sedimentation equilibrium method cannot cope quantitatively with phase coexistence at a first-order phase transition (fluidnematic liquid crystal phase coexistence in the present case).

We now consider the effect of increasing $\beta^{*}$ from zero. All MC simulations were now made for disks with the hard sphere core (needed to avoid electrostatic collapse when 


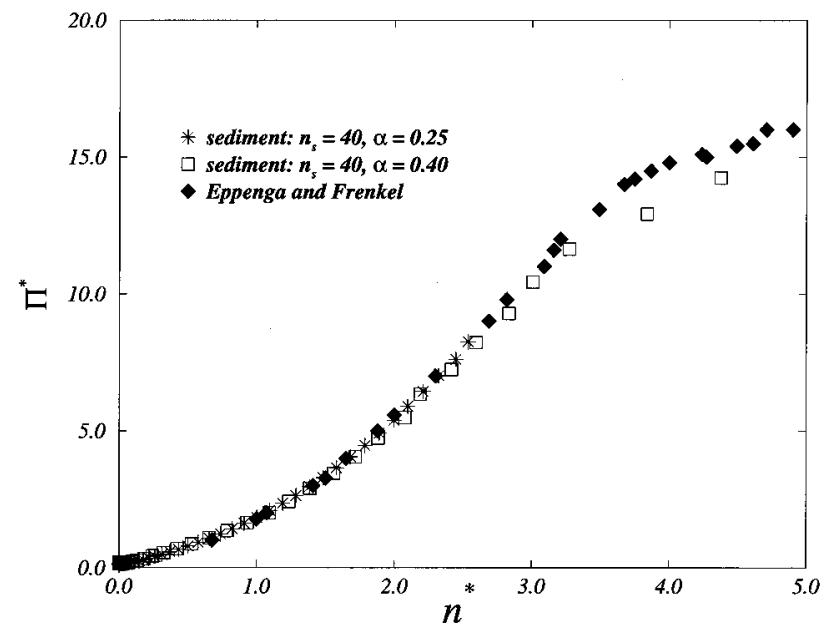

FIG. 1. Reduced osmotic pressure $\Pi^{*}=\Pi \sigma^{3} / k_{B} T$ vs reduced density $n^{*}=N \sigma^{3} / V$ at $\beta^{*}=Q^{2} / \epsilon k_{B} T \sigma^{5}=0$. The stars and squares are the results obtained from simulations of the sedimentation equilibrium for an applied gravitational field of $\alpha=m g \sigma / k_{B} T=0.25$ and 0.40 , respectively. The filled diamonds are the data of Eppenga and Frenkel from Ref. [14].

$\beta^{*} \geqslant 0$ ); when we add the hard sphere core for $\beta=0$, the pressure increases. This pressure difference can be explained by assuming that the equation of state for the infinitely thin hard disks and the hard sphere cores are additive and by using the Carnahan-Starling equation of state for the hard spheres. All profiles were calculated for a fixed value of the reduced density per unit area at the bottom, i.e., for fixed

$$
n_{a}^{*}=n_{a} \sigma^{2}=\sigma^{2} \int_{0}^{\infty} \rho(z) d z
$$

Typical examples are shown in Fig. 2. The profiles are seen to change only slightly from the $\beta^{*}=0$ case, up to $\beta^{*}=0.01$. At $\beta^{*}=0.05$, the profile develops a rounded plateau at the lower altitudes, which becomes very marked and

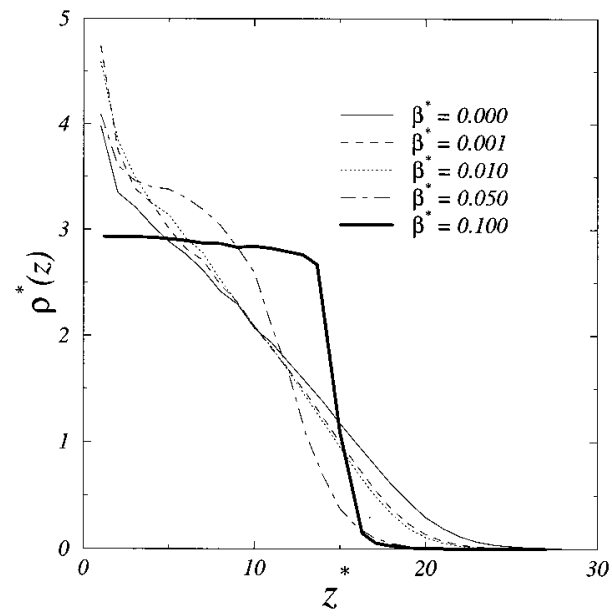

FIG. 2. Density profiles obtained from simulations of the sedimentation equilibrium with an applied gravitational field of $\alpha=m g \sigma / k_{B} T=0.40$. The reduced density $n^{*}(z)=N \sigma^{3} / V$ is plotted vs the height $z^{*}=z / \sigma$ for $\beta^{*}=Q^{2} / \epsilon k_{B} T \sigma^{5}=0.000,0.001$, $0.010,0.050$, and 0.100 .

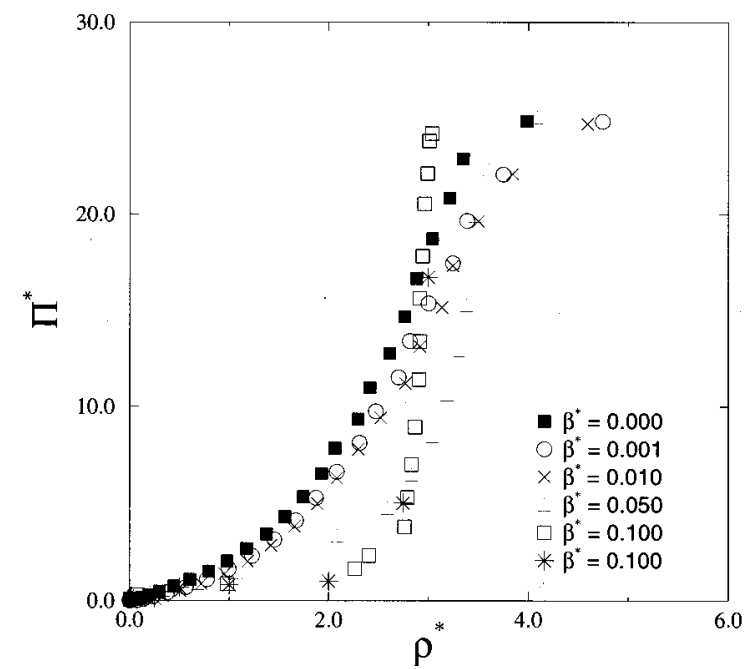

FIG. 3. Reduced osmotic pressure $\Pi^{*}=\Pi \sigma^{3} / k_{B} T$ vs the reduced density $n^{*}=N \sigma^{3} / V$. Filled squares, circles, crosses, triangles, and open squares are the results obtained from the simulations of the sedimentation equilibrium for increasing values of the quadrupolar coupling constant $\beta^{*}=Q^{2} / \epsilon k_{B} T \sigma^{5}$. The stars represent the data from constant temperature-constant density MC simulations at $\beta^{*}=0.1$.

nearly horizontal, followed by an almost discontinuous drop, at $\beta^{*}=0.1$. The resulting equation-of-state data are shown in Fig. 3. For $\beta^{*}=0.001$ and 0.01 the pressures are found to lie slightly below the corresponding values for $\beta^{*}=0$; this reduction may be attributed to dominantly attractive quadrupolar interactions. The isotherms exhibit a clear break beyond $n^{*} \simeq 4$, indicative of the onset of nematic ordering, as observed for $\beta^{*}=0$ by Eppenga and Frenkel [14]. Much more drastic changes are observed for $\beta^{*}=0.05$ and especially for the largest quadrupole moment investigated in our simulations, corresponding to $\beta^{*}=0.1$. In the latter case, $\Pi^{*}$ drops to very low values below $n^{*} \simeq 2.8$, before rising almost vertically. The nearly horizontal portion of the equation of state for $n^{*} \leqslant 2$ is suggestive of a first order phase transition. The steep rise of $\Pi^{*}$ beyond $n^{*} \simeq 2.8$, which contrasts with the much gentler increase at lower values of $\beta^{*}$, shows that the high density phase is nearly incompressible.

In order to obtain a sharper characterization of the alleged phase transition, and of the local structure of the low and high density phases, we have carried out some canonical, constant density (NVT) MC simulations, and Gibbs ensemble calculations [19]. The NVT MC simulations were carried out on samples of $N=250$ disks; they give access to the excess internal energy $U=\left\langle V_{N}\right\rangle$, and to the pressure, which is the sum of the ideal term, of a contact term $\Pi_{c}$, calculated according to the prescription of Ref. [14] and an electrostatic contribution:

$$
\Pi^{*}=\beta \Pi \sigma^{3}=n^{*}+\Pi_{c}^{*}+\frac{5}{3} n^{*} \frac{\beta U}{N}
$$

The excess chemical potential $\mu_{\mathrm{ex}}$ may be estimated by the Widom particle insertion method [20]. A rough estimate of the isothermal compressibility, relative to that of an ideal gas, is obtained by considering the relative fluctuations of the 
TABLE I. The values for the reduced density $n^{*}=N \sigma^{3} / V$, the energy $U^{*}=\beta U / N$, the reduced osmotic pressure $\Pi^{*}=\Pi \sigma^{3} / k_{B} T$, the contact value of the pair distribution function $g(R=\sigma / 2)$, and the density fluctuations $\left(\left\langle N^{2}\right\rangle-\langle N\rangle^{2}\right) /\langle N\rangle$ of the Monte Carlo simulations in the canonical ensemble $(N V T)$ along the isotherm $\beta^{*}=Q^{2} / \epsilon k_{B} T \sigma^{5}=0.1$.

\begin{tabular}{lcccc}
\hline \hline$n^{*}$ & $U^{*}$ & $\Pi^{*}$ & $g(R=\sigma / 2)$ & $\left(\left\langle N^{2}\right\rangle-\langle N\rangle^{2}\right) /\langle N\rangle$ \\
\hline 0.25 & -9.78 & 0.12 & 30 & 5.6 \\
0.5 & -9.62 & 0.67 & 25 & 3.1 \\
1.0 & -11.20 & 0.81 & 25 & 1.6 \\
2.0 & -12.26 & 1.01 & 10 & 1.3 \\
3.0 & -12.32 & 16.70 & 7.0 & $\simeq 0.0$ \\
5.5 & -12.22 & 43.38 & 7.0 & $\simeq 0.0$ \\
\hline
\end{tabular}

number of particles within subvolumes equal to one-eighth of the total volume. The resulting data along the isotherm $\beta^{*}=0.1$ are listed in Table I. As shown in Fig. 1 , the $N V T$ $\mathrm{MC}$ results for the pressure $\Pi$ agree very well with the equation of state previously determined by the sedimentation equilibrium method. The MC simulations also yield structural information on the positional and orientational shortrange order in the suspension. Snapshots of typical configurations along the isotherm $\beta^{*}=0.1$ are shown in Figs. 4(a) and 4(b) for $n^{*}=0.25,3$, and 5.5. Figures 5(a) and 5(b) show histograms of the distribution $f(R, \theta)$ of the angles between the orientation vectors of pairs of particles separated by a distance $R \leqslant 3 \sigma$. Complete information on the pair structure is contained in the full pair distribution function $g\left(\vec{R}, \Omega_{1}, \Omega_{2}\right)$, which may be expanded into rotational invariants, with coefficients $g^{l_{1}, l_{2}, l}(R)[13,14] . g(R)=g^{000}(R)$ is the usual pair distribution function of center-to-center distances. An example is shown in Fig. 6; the general shape of $g(R)$ changes remarkably little with density, except the value of closest approach, corresponding to the hard-core cutoff at $R=\sigma / 2$, which increases as $n^{*}$ decreases [approximate values of $g(R=\sigma / 2)$ are listed in Table I]. Integration of $g(R)$ up to the first minimum beyond the main peak yields an estimate of the number of nearest neighbors $\nu$ (first coordination shell) close to 3 , and practically independent of density up to $n^{*}=3$.

Orientational correlations are best characterized by

$$
\begin{aligned}
g_{\text {or }}(R) & =g^{202}(R)=\left\langle P_{2}[\cos \theta(R)]\right\rangle=\left\langle\frac{1}{2}\left[3 \cos ^{2} \theta(R)-1\right]\right\rangle \\
& =\int_{0}^{\pi} \frac{1}{2}\left[3 \cos ^{2} \theta-1\right] f(R, \theta) \sin \theta d \theta
\end{aligned}
$$

where the distribution function $f$ is assumed to be normalized to 1 for each distance $R$. Examples of $g_{\text {or }}(R)$ are shown in Figs. 7(a) and 7(b) and 8(a)-8(c). Along the isotherm $\beta^{*}=0.1, g_{\text {or }}(R)$ barely changes up to $n^{*} \simeq 3$; in particular the function goes rapidly to zero beyond $R \simeq 2 \sigma$, underlining the complete absence of any long-range order in the orientational correlations. The situation changes for $n^{*} \geqslant 3$ as shown in Fig. 8; at these higher densities, $g_{\text {or }}(R)$ is seen to go to a nonzero asymptotic value characteristic of long-range nematic ordering. The usual nematic order parameter $S=\left\langle P_{2}\left(\cos \theta_{i}\right)\right\rangle$ is related to this asymptotic value by
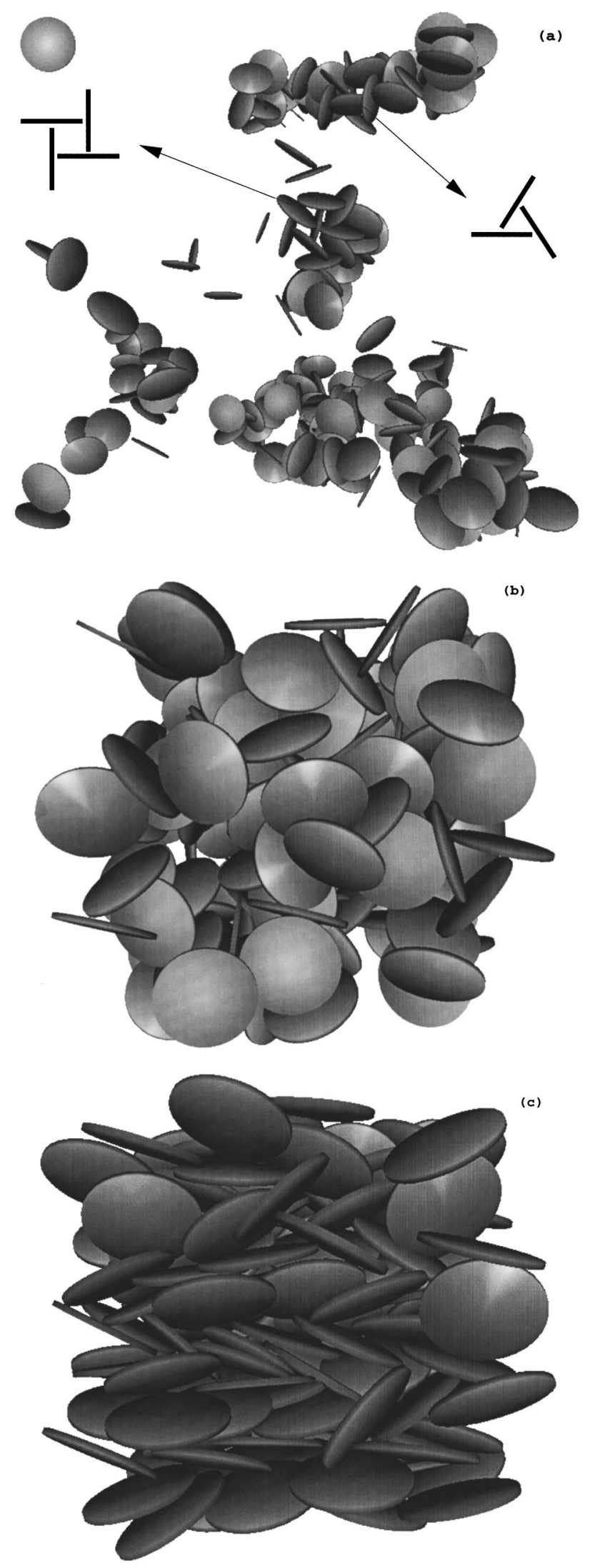

FIG. 4. Snapshots of typical configurations of quadrupolar disks are shown obtained from constant temperature-constant density simulations at $\beta^{*}=Q^{2} / \epsilon k_{B} T \sigma^{5}=0.1$ for several reduced density $n^{*}=N \sigma^{3} / V$ : (a) $n^{*}=0.25$, (b) $n^{*}=3.0$, (c) $n^{*}=5.5$. 

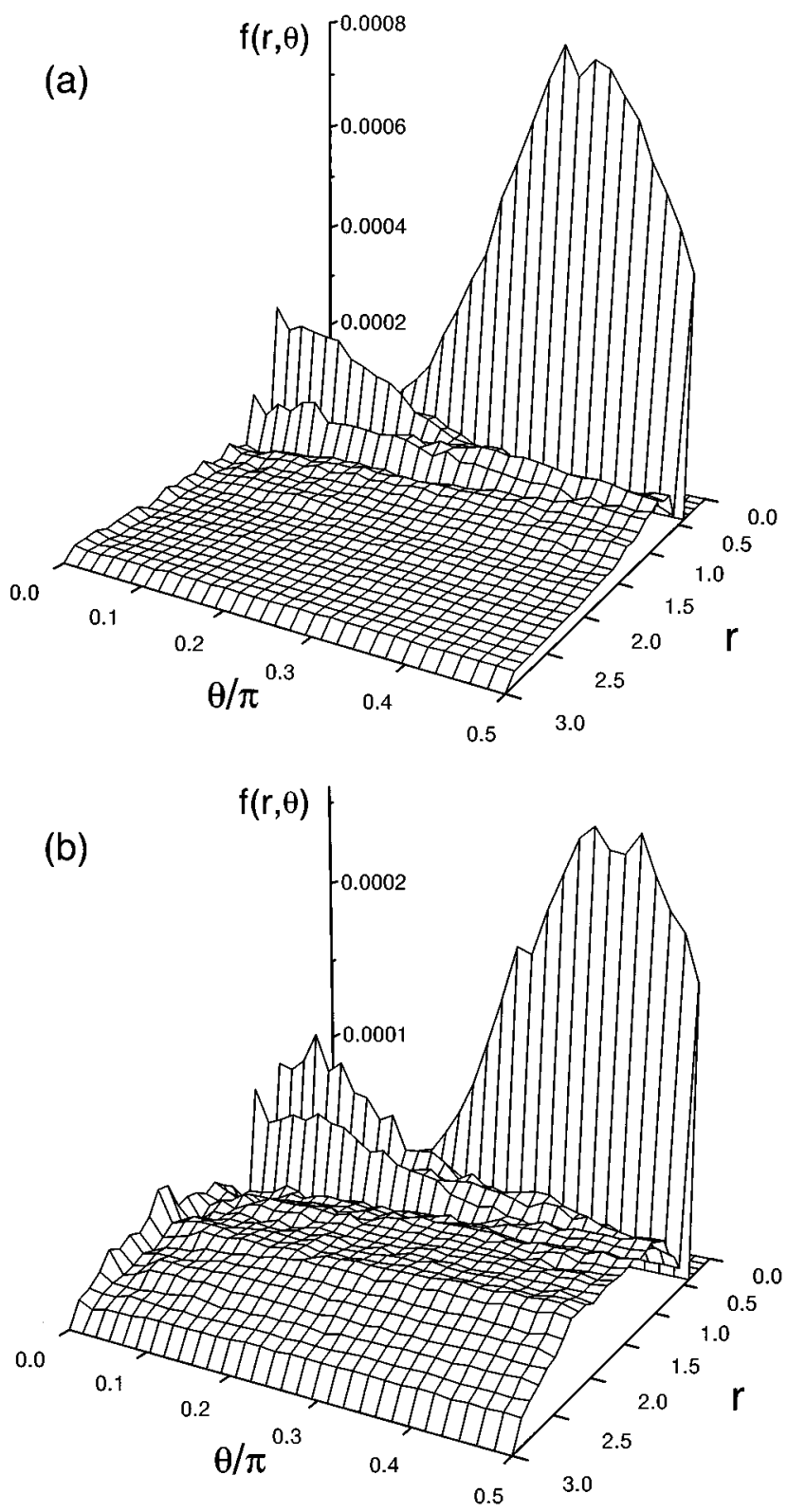

FIG. 5. Histograms of the distribution $f(R, \theta)$ of the angles between the orientation vectors of pairs of particles separated by a distance $R \leqslant 3 \sigma$ at reduced density $n^{*}=N \sigma^{3} / V$. (a) $n^{*}=0.25$, (b) $n^{*}=2.0$.

$$
\lim _{R \rightarrow \infty} g_{\text {or }}(R)=S^{2}
$$

An even more dramatic change is observed when the coupling constant $\beta^{*}$ is lowered from 0.1 to 0.01 (i.e., as the temperature increases) along the isochore $n^{*}=5.5$, as is evident from Figs. 8(b) and 8(c). In the next section these MC data are analyzed and interpreted in the light of a sol-gel transition scenario.

\section{A REVERSIBLE SOL-GEL TRANSITION}

Inspection of Figs. 2 and 3 suggests that the quadrupolar interactions between disks do not induce any major changes compared to the behavior of bare $(Q=0)$ disks, at least up to $\beta^{*}=0.01$. The electrostatic interactions lead only to a slight

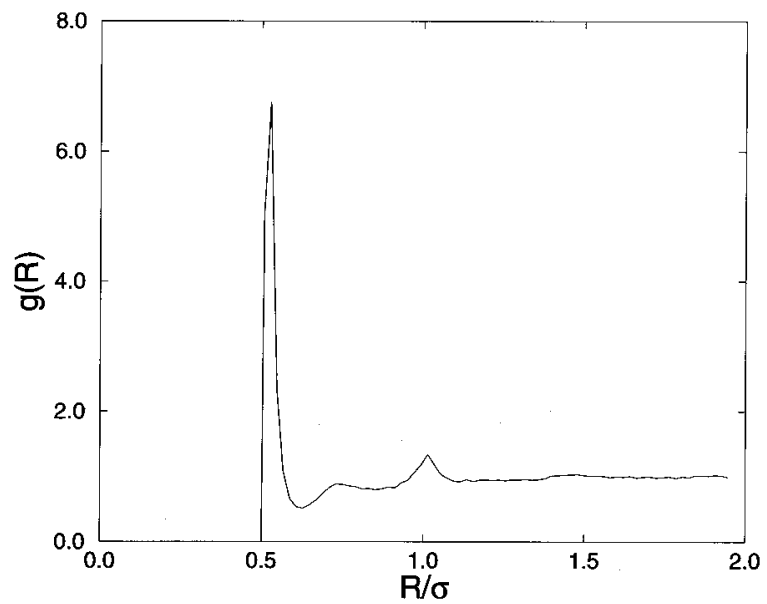

FIG. 6. The pair distribution function of center-to-center distances $g(R)$ is plotted for a quadrupolar coupling constant $\beta^{*}=Q^{2} / \epsilon k_{B} T \sigma^{5}=0.1$ and reduced density $n^{*}=N \sigma^{3} / V=3$.

lowering of the osmotic pressure. Although we have not investigated this point in detail, the break in the equations of state beyond $n^{*} \simeq 4$, as well as the orientational distribution function in Fig. 8(c), where the large positive value of $g_{\text {or }}(R)$ at $R=\sigma / 2$ points to parallel ordering of nearest neighbors, which propagates to large distances, strongly suggest that the isotropic-to-nematic phase transition reported by Ep-
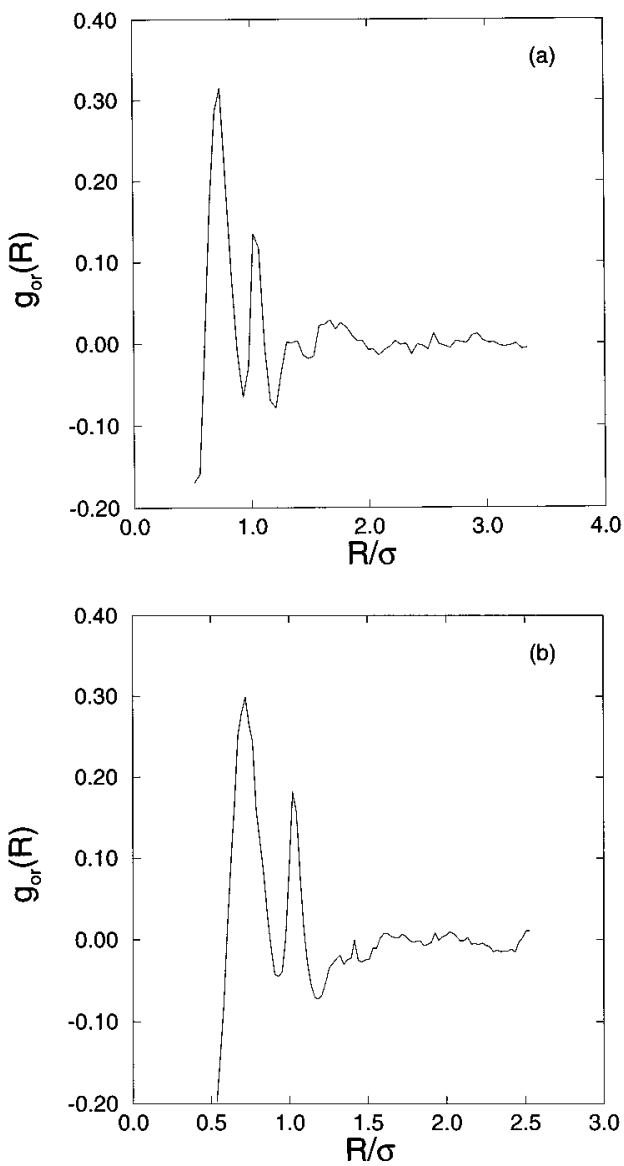

FIG. 7. The orientational pair distribution function $g_{\text {or }}(R)$ is plotted for a quadrupolar coupling constant $\beta^{*}=Q^{2} / \epsilon k_{B} T \sigma^{5}=0.1$ and reduced density $n^{*}=N \sigma^{3} / V$ : (a) $n^{*}=0.25$, (b) $n^{*}=2.0$. 

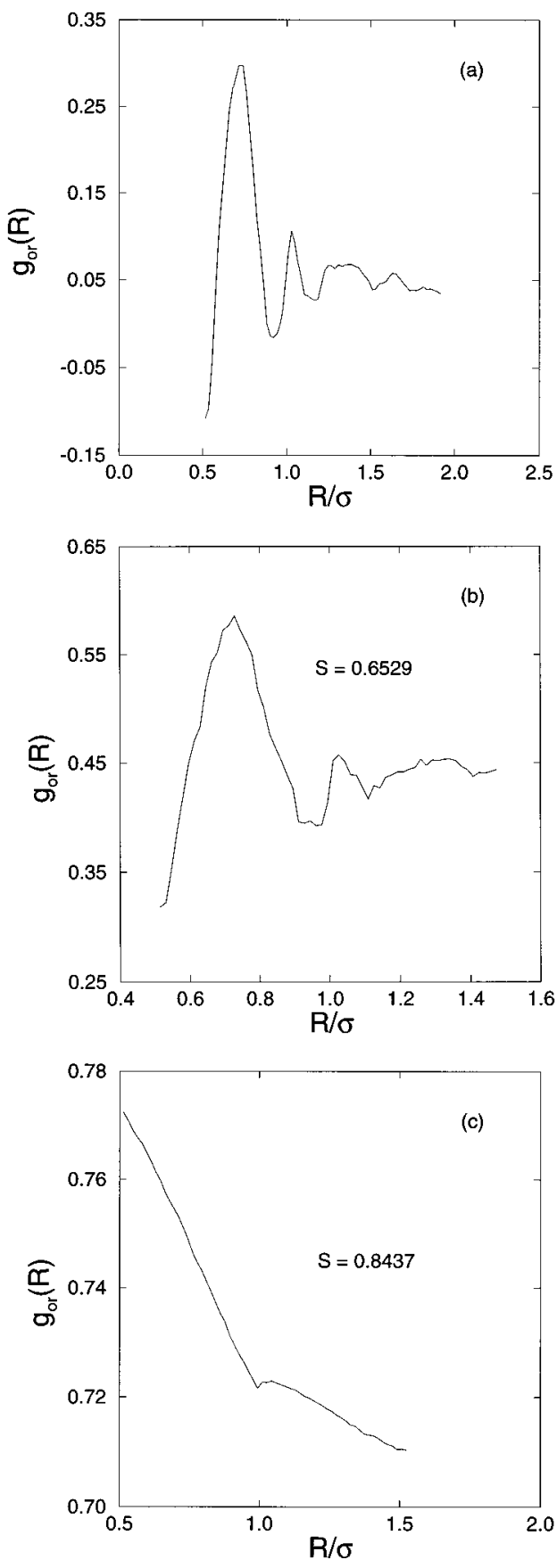

FIG. 8. The orientational pair distribution function $g_{\text {or }}(R)$ is plotted for several values for $\beta^{*}=Q^{2} / \epsilon k_{B} T \sigma^{5}$ and $n^{*}=N \sigma^{3} / V$ : (a) $\beta^{*}=0.1, n^{*}=3$, (b) $\beta^{*}=0.1, n^{*}=5.5$, (c) $\beta^{*}=0.01, n^{*}=5.5$.

penga and Frenkel for $\beta^{*}=0$ [14] is very little affected by the electrostatic interactions at high temperatures, or equivalently at low values of the quadrupole moment $Q$.

This simple behavior changes at higher values of $\beta^{*}$. We have explored in some detail the isotherm $\beta^{*}=0.1$. For $n^{*} \leqslant 2$, the osmotic pressures are now very low compared to the bare disk case ( $Q=0$ limit). Despite the relatively large statistical uncertainties (essentially linked to the difficulties of extracting accurate contact values of the pair distribution function in the NVT MC simulations), the data listed in Table I suggest a roughly constant value of $\Pi^{*}$ for $0.5 \leqslant n^{*} \leqslant 2$, which points to a horizontal tie-line indicative of a first-order phase transition. The snapshots in Fig. 4 show that the low-density configurations are highly inhomogeneous: the quadrupolar disks form elongated clusters dominated by edge-to-face arrangements of nearest neighbors. The clusters are somewhat reminiscent of "living" polymers since they continuously break up and recombine in the coarse of a simulation, thus ensuring an adequate exploration of configuration space.

The pattern of orientational correlations, embodied in the histograms $f(R, \theta)$ in Fig. 5, and its projection $g_{\text {or }}(R)$ in Fig. 7 , is very striking. The histograms show a broad peak at angles $\theta$ close to $\pi / 2$ and $R=0.5 \sigma$, which reflects itself in the negative value of $g_{\text {or }}(R)$ at contact. This feature is a clear signature of the dominant edge-to-face pair configurations, with angles $\pi / 3 \leqslant \theta \leqslant \pi / 2$. The histograms and orientational distribution functions furthermore show that disks are mainly parallel to each other $(\theta \simeq 0)$ for distances $R / \sigma \simeq \sqrt{2} / 2$ and $R / \sigma \simeq 1$, as expected from a four-particle ring [schematically shown in Fig. 4(a)] and from an $\mathrm{H}$-like configuration (where two parallel disks are separated by a third disk in contact with and orthogonal to the former). The histograms and $g_{\text {or }}(R)$ show very little structure beyond $R / \sigma \simeq 1.5$, at all densities $n^{*} \leqslant 3$.

The representative configuration of the dense state $n^{*}=3$ [Fig. 4(b)] is very reminiscent of the "house-ofcards" structure frequently advocated as being typical of clay colloid gels [6]. The clusters of the low concentration suspensions have now merged into a space-filling (or percolating) network, which is, however, still characterized by the same orientational correlations, as illustrated by the great similarity between the orientational correlation functions $g_{\text {or }}(R)$ in Figs. 7(a) and 8(a). The network is highly incompressible, as shown by the dramatic, nearly vertical increase in pressure around $n^{*} \simeq 2.8$, where the reduced isothermal compressibility $\chi_{T}^{*}=\partial n^{*} / \partial \Pi^{*}$ is estimated to be less than 0.01 . The resistance of the "house-of-cards" network to compression is due to the strong electrostatic bias towards edge-to-face pair configurations, which are clearly evident in Fig. 4(b). However, at sufficiently high pressure, the edgeto-face electrostatic cross linking may be broken, and parallel stacking predominates, as shown in the configuration of Fig. 4(c) for $n^{*}=5.5$. As confirmed by the corresponding $g_{\text {or }}(R)$ pictured in Fig. 8(b), the T-shaped configurations (signalled by negative values of $g_{\text {or }}(R \simeq \sigma / 2)$ at lower densities) have been replaced by predominantly parallel alignment of neighboring platelets, which propagates to large distances $R$, giving rise to the long-range order characteristic of a nematic phase.

Returning to the density range $0.25 \leqslant n^{*} \leqslant 3$, the data lead us to conjecture about the existence of a first-order phase transition between a low density sol phase, where the suspended platelets form well-separated clusters, and a high density gel phase, where the platelets are arranged in a space-filling network with predominantly edge-to-face crosslinking involving "binding" energies of several times the thermal energy $k_{B} T$.

In order to confirm this conjecture we have carried out Gibbs-ensemble MC simulations [19] to determine the coexistence curve between the low and high density phases accurately. In this method the two phases are placed in separate simulation cells which may exchange volume and particles at a given temperature (i.e., $\beta^{*}$ ) in order to fulfill the phase 


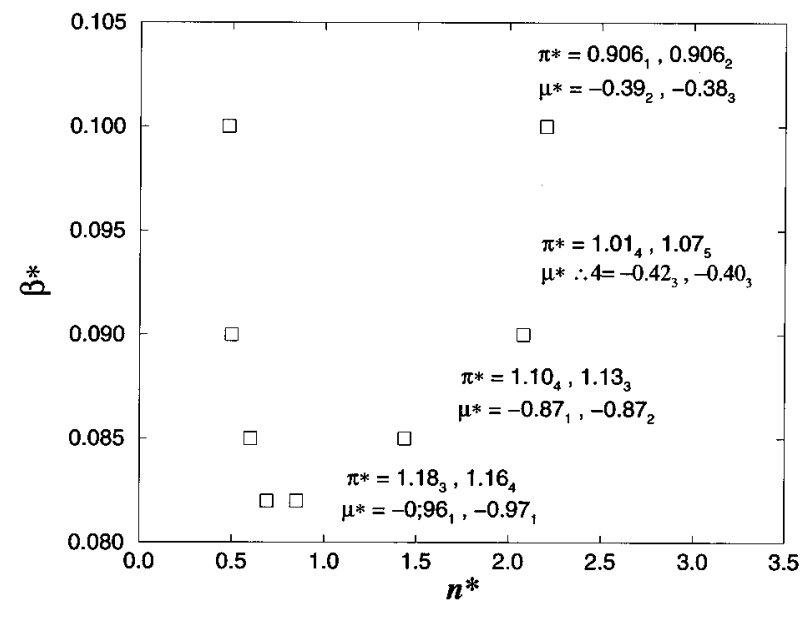

FIG. 9. Sol-gel coexistence curve obtained from Gibbsensemble MC simulations in the quadrupolar coupling constant $\beta^{*}=Q^{2} / \epsilon k_{B} T \sigma^{5}$ and reduced density $n^{*}=N \sigma^{3} / V$ plane.

equilibrium requirements of equal pressures and chemical potentials. Calculations were carried out for $\beta^{*}=0.10,0.09$, 0.085 , and 0.0825 , and the resulting phase diagram is plotted in Fig. 9. The critical coupling constant $\beta^{*}$, above which a reversible sol-gel phase separation occurs, may be estimated to be $\beta_{c}^{*}=0.082$, while the corresponding critical density is roughly $n_{c}^{*}=0.75$. Along the isotherm $\beta^{*}=0.1$, the densities of the coexisting phases are predicted to be $n^{*} \simeq 0.48$ and 2.2 , in rough agreement with the end points of the "horizontal" tie line in the equation of state shown in Fig. 2. The states at intermediate densities studied in the NVT MC simulations discussed earlier are thus expected to be metastable (or possibly unstable) in the thermodynamic limit, but are artificially stabilized by finite-size effects.

The good convergence of the Gibbs ensemble MC simulations shows that the sol-gel transition observed for quadrupolar disks is a genuine, reversible first-order phase transition, reminiscent of a gas-liquid transition in ordinary atomic or molecular fluids. The phase diagram in Fig. 9 is incomplete, in that we have not attempted to map out the transition line from the isotropic gel phase to the anisotropic nematic phase at higher densities $\left(n^{*} \geqslant 4\right)$. Below $\beta^{*} \simeq 0.082$ this is the only phase transition to be expected for the quadrupolar disk model. As expected, the phase diagram of quadrupolar disks has little in common with the fluid-solid phase diagram of quadrupolar hard spheres, as determined in recent $\mathrm{MC}$ simulations [21].

\section{DISCUSSION}

Starting from the physical charcteristic of a real clay colloid suspension (Laponite RD), we have conducted two successive reduction steps to map the initial system onto a model of nonintersecting disks carrying a fixed quadrupole moment. In the process of the first step, we have neglected the discrete nature of the suspending fluid (water) and all interactions between polyions, except those of steric and electrostatic origin, ending up at the level of a "primitive", model of clay suspensions. In particular, we have at this stage neglected the ubiquitous van der Waals attractions, as well as any hydration or depletion effects. Such interactions could lead to quantitative and even qualitative changes in the structure and phase behavior of real clay suspension, compared to the predictions of the "primitive" model.

We have sketched a possible treatment of the latter via density functional theory, along the lines of Ref. [9], but in view of the remaining severe technical difficulties, linked to the anisometry of the charged clay platelets, we have been led to a second reductive step, whereby each platelet and its associated electric double layer, due to the buildup of an inhomogeneous and anisotropic charge distribution of coions and counterions, is replaced by a disk carrying a rigid point quadrupole. This is clearly a drastic oversimplification of the original electrostatic problem posed by the "primitive", model, since it totaly ignores fluctuation and interference effects of interpenetrating electric double layers, which lead to configuration-dependent many-body interactions between the polyions [9]. Thus the quadrupolar disk model that we have examined in this paper can be regarded, at best, as a crude representation of a suspension of Laponite clay particles. Even if the configuration dependence of the effective quadrupole moment $Q$ is neglected, it should depend on the concentration of added salt, and on the polyion concentration [12]. The latter effect is also neglected in the present model, while the salt concentration $n_{s}$, together with the temperature, determine the quadrupolar coupling constant $\beta^{*}$.

It is instructive to compare the phase behavior of the present schematic model with experiments on Laponite suspensions. The experiments in Ref. [1] also report a reversible sol-gel transition for monovalent salt concentrations less than $10^{-2} M$ (at higher salt concentration, the suspension is observed to flocculate). The most surprising finding of Mourchid et al. [1] is the shift of the sol-gel transition to lower clay concentrations with increasing salt concentration. This feature cannot be understood in the framework of the present model, since an increase in salt concentration invariably leads to a reduction of $Q$ and hence of $\beta^{*}$. For a $10^{-4} M$ salt concentration, the experiments detect gelation at a Laponite concentration of about $3 \%$ by weight. With a polyion diameter of $25 \mathrm{~nm}$ and a charge $Z=700$, these conditions correspond to $n^{*} \simeq 0.37$, and a ratio $\alpha=Q_{\text {total }} / Q_{d} \simeq 0.035$, such that $\beta^{*} \simeq 0.065$ at room temperature in water; these values are fairly close to the critical point coordinates reported here for the quadrupolar disk model.

The traditional picture of a "house-of-cards" structure has been questioned in the light of available experimental data on Laponite [1-3], although there is no consensus [4]. The quadrupolar disk model, considered in the present work, gives rise to a network structure in the gel phase strongly reminiscent of a "house-of-cards." In view of the considerable simplifications that have to be made to arrive at this model, our results should not be regarded as contradicting experimental evidence. On the contrary, it would be instructive to understand how the physical aspects neglected in our model, such as van der Waals attractions or double-layer fluctuations and interference effects, might transform the present gel structure into the heterogeneous stackings that have been imagined to interpret experimental data.

\section{ACKNOWLEDGMENTS}

We gratefully acknowledge stimulating discussion with M. Kroon, G. Wegdam, E. Trizac, and A. Delville and cor- 
respondence with H.N.W. Lekkerkerker, who pointed out Ref. [1]. The work was supported by the European Commu- nity, the EPSRC, and the Ministere des Affaires Etrangeres (France) and the British Council.
[1] A. Mourchid, A. Delville, J. Lambard, E. Lecolier, and P. Levitz, Langmuir 11, 1942 (1995).

[2] J.D.F. Ramsay, S.W. Swanton, and J. Bruce, J. Chem. Soc. Faraday Trans. 86, 3919 (1990).

[3] M. Morvan, D. Espinat, J. Lambard, and Th. Zemb, Colloids and Surfaces A 82, 193 (1994).

[4] S.D.T. Axford and T.M. Herrington, J. Chem. Soc. Faraday Trans. 90, 2085 (1994).

[5] M. Kroon, G.H. Wegdam, and R. Sprik, Europhys. Lett. 35, 621 (1996).

[6] H. van Olphen, Clay Colloid Chemistry, 2nd ed. (Wiley, New York, 1977).

[7] M. Dijkstra, J.P. Hansen, and P.A. Madden, Phys. Rev. Lett. 75, 2236 (1995).

[8] For recent reviews, see R. Evans, in Fundamentals of Inhomogeneous Fluids, edited by D. Henderson (Marcel Dekker, New York, 1992); or J.P. Hansen, in Phase Transitions in Complex Fluids, edited by M. Baus, L.P. Rull, and J.P. Rijckaert (Kluwer, Dordrecht, 1994).

[9] H. Löwen, J.P. Hansen, and P.A. Madden, J. Chem. Phys. 98, 3275 (1993).
[10] J.P. Hansen and E. Smargiassi, in Monte Carlo and Molecular Dynamics of Condensed Matter Systems, edited by K. Binder and G. Ciccotti (SIF, Bologna, 1995).

[11] R. Car and M. Parrinello, Phys. Rev. Lett. 55, 2471 (1986). For a review see D.K. Remler and P.A. Madden, Mol. Phys. 70, 921 (1990).

[12] J.P. Hansen and E. Trizac, Physica A (to be published).

[13] L. Blum and A.J. Torruella, J. Chem. Phys. 56, 303 (1971).

[14] R. Eppenga and D. Frenkel, Mol. Phys. 52, 1303 (1984).

[15] G.J. Zarragoicoechea, D. Levesque, and J.J. Weis, Mol. Phys. 78, 1475 (1993).

[16] S. Hachisu and K. Takano, Adv. Colloid Interface Sci. 16, 233 (1982).

[17] T. Biben, J.P. Hansen, and J.L. Barrat, J. Chem. Phys. 98, 7330 (1993).

[18] R. Piazza, T. Bellini, and V. Degiorgo, Phys. Rev. Lett. 71, 4267 (1993).

[19] A.Z. Panagiotopoulos, Mol. Phys. 61, 813 (1987).

[20] B. Widom, J. Chem. Phys. 39, 2808 (1963).

[21] C. Vega and P.A. Monson, J. Chem. Phys. 102, 1361 (1995). 